\title{
Criminologie
}

\section{Résurgence du mouvement antiprohibitionniste}

\section{Marie-Andrée Bertrand}

Volume 22, numéro 1, 1989

Drogues et criminalités

URI : https://id.erudit.org/iderudit/017277ar

DOI : https://doi.org/10.7202/017277ar

Aller au sommaire du numéro

Éditeur(s)

Les Presses de l'Université de Montréal

ISSN

0316-0041 (imprimé)

1492-1367 (numérique)

Découvrir la revue

Citer cet article

Bertrand, M.-A. (1989). Résurgence du mouvement antiprohibitionniste.

Criminologie, 22(1), 121-133. https://doi.org/10.7202/017277ar

\section{Résumé de l'article}

Recently, one could witness a resurgence of the debate on the drug policy, a debate that many thought passé since the late seventies. Adding to the already existing arguments against the international conventions and the national drug laws the negative experience of 15 years of repression and the fait accompli of the lost "war against drugs", the new movement, an international one, has become openly anti-prohibitionist. 
Recently, one could witness a resurgence of the debate on the drug policy, a debate that many thought passé since the late seventies. Adding to the already existing arguments against the international conventions and the national drug laws the negative experience of 15 years of repression and the fait accompli of the lost «war against drugs», the new movement, an international one, has become openly anti-prohibitionist.

\section{L'ACTUALITÉ DU DÉBAT}

Plusieurs observateurs de la scène canadienne et américaine ont pu croire, avec raison, que le débat sur la question de la politique des drogues s'était éteint avec les années 70 : à part quelques timides projets de loi vite enterrés aux feuilletons des parlements ou quelques pratiques décriminalisantes appliquées sur des bases locales, on ne voyait guère que la National Organization for the Repeal of Marijuana Laws (et encore les activités de cette organisation n'étaient-elles pas connues du grand public) soucieuse de raviver le feu, à la toute fin des années 70 .

Pourtant, le débat n'était pas mort. Ou s'il l'était, il faut maintenant constater qu'il reprend vie depuis le début des années 80 .

Trois faits en témoignent, qui ont une portée internationale :

1. en Europe s'est créé, il y a quelques années, le Mouvement européen pour la normalisation des politiques sur les drogues regroupant, en particulier, des Hollandais, des Suisses, des Danois, des Britanniques, un mouvement qui s'est efforcé d'influencer les politiques internationales élaborées par les nombreux organes et agences des Nations-Unies;

2. en Europe également est née, sous l'égide du Parti radical italien, CO.R.A., c'est-à-dire la Coordination radicale antiprohibitionniste. Celle-ci organisait, à Bruxelles, en octobre 1988, un colloque international portant carrément sur l'antiprohibitionnisme, regroupant une quarantaine des chercheurs, des personnalités politiques et des gens d'action de la majorité des pays européens, ainsi que du Canada, des États-Unis et de l'Australie;

3. aux États-Unis est née récemment la Fondation pour la poli-

* Marie Andrée Bertrand, professeure titulaire, École de criminologie, Université de Montréal, C.P. 6128, Succursale A, Montréal, Québec, H3C 3J7. 
tique des drogues qui a tenu une première conférence internationale à Londres en 1987 et une deuxième à Washington en octobre 1988 sur le thème: Practical Models of Peaceful Drug Policy Reform. Les réunions de la Drug Policy Foundation attirent les principaux représentants des deux organismes européens précités ainsi que des participants d'Australie, du Canada, d'Amérique latine, sans compter évidemment de nombreux experts et personnalités politiques des États-Unis, soit une centaine de participants dont plusieurs ont une réputation internationale.

Nous décrirons plus loin les solutions de rechange proposées par ces trois organismes à la politique pénale présentement imposée par les Nations-Unies.

Aux indices de la reprise du débat sur la politique des drogues que constituent ces réunions d'experts et de gens d'action, ajoutons d'autres faits plus susceptibles d'avoir rejoint le grand public. Par exemple, en mai 1988, le magazine Time titrait, en page couverture: «Should drugs be made legal?» et dans l'ensemble de ce numéro (30 mai), on posait la question de la politique des drogues en des termes plus radicaux que ceux des années 1960 ou 1970 : c'est en général, l'échec de la «War on drugs» qui est alors décrit et analysé, par des hommes politiques (sénateurs, maires) et des experts, y compris des spécialistes de la politique pénale. Les candidats à la présidence ont fait une large place à la question dans leur campagne pré-électorale. Plus près de nous, au Canada, la radio et la télévision font à nouveau une place à la politique des drogues, y consacrant un nombre d'heures non négligeable.

L'actualité du débat autorise à en reprendre les arguments déjà évoqués en 1970-75, mais auxquels s'en sont ajoutés de nouveaux, plus convaincants et à saveur plus définitive.

\section{BREF RAPPEL DES ARGUMENTS ANTI-PROHIBITIONNISTES 1}

Lorsque, il y a quinze ans, au terme de quatre années d'études et d'enquêtes à travers tout le pays et à l'étranger, j'ai présenté au gouvernement canadien un rapport minoritaire réclamant l'abolition du contrôle pénal sur les drogues, j'étais motivée par les raisons suivantes:

1. le délit de simple possession est un moyen futile de dissuasion;

1. Les points 2,3 et 4 du présent article, soit: le rappel des arguments militant contre la prohibition et l'analyse des résistances, ont fait l'objet de la communication présentée par l'auteure au Colloque international sur l'Anti-prohibitionnisme qui s'est tenu à Bruxelles, du 29 septembre au 2 octobre 1988, sous l'égide du Parti radical. 
2. plus généralement, l'utilisation du droit pénal contre les crimes sans victimes a) est inefficace; b) entraîne le recours à des procédés qui vont contre le droit des personnes: fouilles, perquisitions sans mandat, délateurs, agents doubles; c) est toujours hautement arbitraire puisque les moyens de détection habituels font défaut et que seule une frange naïve ou démunie est sujette à la répression.

Nombreux d'ailleurs sont les philosophes du Droit et les hommes d'état qui ont reconnu que le recours au droit criminel pour réduire les «crimes sans victimes» est illégitime;

3. les coûts de la prohibition sont énormes: coût sociaux, moraux économiques; les états y gaspillent leur honneur, les fonds publics (Hulsman, 1983) et ces coûts sont sans proportion avec l'efficacité improbable et minimale de la loi (Le Dain, 1973, 109);

4. la fonction pédagogique du droit pénal, qui doit rappeler aux citoyens les valeurs les plus chères au groupe social, se trouve pervertie par l'inclusion, pêle-mêle, dans une même loi, de substances de nocivité très variable et de comportements de gravité fort différente. En effet, les statuts sur les drogues prévoient encore, dans plusieurs pays, des peines sévères allant jusqu'à l'emprisonnement pour des actes sans réelle gravité et qui ne nuisent pas à autrui et pour la possession et le trafic de substances sans grande nocivité avérée; de plus, les états invoquent leur devoir de protéger la santé des citoyens pour fonder le contrôle pénal de certaines substances en même temps qu'ils touchent des revenus considérables de la vente d'autres drogues jugées tout à fait nocives comme le tabac et l'alcool;

5. la prohibition crée et fait proliférer les marchés illicites et tous les vices qui y sont attachés;

6. le délit de simple possession ou d'usage, comme en France par exemple, autorise certains états à recourir au traitement obligatoire à l'égard des accusés, autre violation des droits de la personne;

7. les prisons et pénitenciers où sont détenus un certain nombre d'usagers regorgent de substances psychothropes de toutes natures et il s'y pratique un trafic aussi important sinon plus important que celui qui a cours dans le monde libre.

Tous ces arguments militant en faveur de l'abolition des lois sur les drogues sont encore valides. Bien plus, les années en ont accru la portée. En effet, et en les reprenant un à un, on voit que :

1. l'effet de dissuasion s'est avéré nul, sinon sur quelques usagers en particulier, du moins sur l'ensemble des populations visées. Le nombre des usagers a augmenté, le trafic s'est mieux organisé; il se 
peut que la consommation de certaines drogues populaires dans les années 60 et 70 ait décru dans les années 80 (le LSD, la colle, les hallucinogènes puissants) (Blackwell, 1988, 238-241) mais nous savons tous que le droit pénal n'y est pour rien. Ce sont les usagers eux-mêmes, l'opinion publique et l'absence de profits avantageux qui ont contribué à ce déclin comme le montrent les études portant sur la consommation dans les états américains qui ont pratiqué la dépénalisation (Single, 1988);

2. le contrôle pénal de l'usage et du trafic s'est révélé hautement discriminatoire, frappant d'abord, dans les années 60 , les jeunes et les personnes à l'apparence non conformiste; puis les membres des minorités ethniques, et récemment les étrangers dans plusieurs pays européens, ainsi que les pauvres et les sans emploi;

3. le coût de l'application des lois sur les drogues n'a cessé de grimper; ces lois ont entraîné la création de services spéciaux de police, ont surchargé les tribunaux, les prisons, les services de cure et de postcure pénale;

4. les prophéties épidémiologiques se sont avérées sans fondement; certes, il y a des toxicomanes qui ont besoin d'aide mais les urgences des hopitaux sont moins encombrées qu'elles ne l'étaient dans les années 70 par les patients souffrant des effets d'intoxications aux drogues illicites (Negrete, 1988);

5. la politique prohibitionniste a, et comme prévu, fait se développer les marchés illicites; le commerce international s'intensifie mais les corps policiers de tous les pays ont perdu la guerre contre la drogue et tous avouent ne contrôler qu'à peine $10 \%$ des importations;

6. le traitement non volontaire et l'emprisonnement des toxicomanes pour réduire leur habitude se sont soldés par des échecs retentissants.

D'autre part, des faits nouveaux sont venus ajouter aux raisons qu'on avait, il y a quinze ans, de recommander l'abolition des lois sur les drogues.

1. Dans plusieurs pays occidentaux, on est arrivé à la conclusion que plusieurs drogues licites causent un tort certain à la santé des citoyens et que les coûts entraînés par l'abus de ces substances dépassent le seuil tolérable. Cette constatation a deux effets. Premièrement, elle montre bien l'incohérence des états qui se préoccupent de quelques substances pour en faire l'objet d'interdiction dans le code pénal mais en même temps encouragent secrètement ou publiquement la consom- 
mation de nicotine et d'alcool; deuxièmement, les réactions sociales aux méfaits de ces deux drogues s'avèrent beaucoup plus saines et efficaces que celles qui prévalent dans le domaine des drogues illicites. Les états, certains en tout cas, interdisent sur les ondes de la radio et de la télé d'état, la promotion de ces substances ou exigent qu'on assortisse cette publicité de mises en garde; le public et les associations de citoyens se mobilisent pour limiter les méfaits de la consommation aux seuls usagers dans le cas de la cigarette. Touchant l'alcool, la réaction est aussi intéressante. Puisque c'est la conduite en état d'ébriété qui constitue un danger social, c'est à cela qu'on s'attaque et non à la consommation; des campagnes intelligentes permettent à l'usager de consommer sans mettre la vie des autres citoyens en danger (l'opération Nez Rouge, par exemple). Là non plus, cependant, le recours à la seule solution pénale ne constitue pas une réponse adéquate.

2. Comme tant d'autres, j'ai été frappée par la montagne de résistances à laquelle se sont heurtées les conclusions et recommandations de la plupart des commissions d'enquête, des différents pays, sur la question des drogues. En effet, dans les années 70, pas moins d'une douzaine de pays avaient procédé, par l'intermédiaire de comités nationaux ou de commissions d'enquête, à l'examen de ce qu'on appelait alors «le problème de la drogue»: son étendue, ses causes, les moyens d'y porter remède. Aucun de ces comités, aucune de ces commissions, sauf peut-être le Comité Pelletier en France, n'avait recommandé à son Parlement de s'en tenir au statu quo. L'un plaidait pour la décriminalisation de certaines substances, l'autre pour l'abolition du crime de simple possession, etc.

C'est en analysant les sources de ces résistances au changement des loi sur les drogues que me sont apparues clairement l'immoralité, l'hypocrisie et l'illégitimité de la prohibition.

Ces résistances se situent au niveau national et au niveau international.

\section{LES RÉSISTANCES AU NIVEAU NATIONAL À L'ABOLITION DE LA PROHIBITION}

Les recommandations des commissions nationales étaient bien rarement ou à peu près jamais radicales mais malgré cela les parlements les ont souvent ignorées ou diluées jusqu'à les rendre à peu près ridicules ou méconnaissables sauf aux Pays-Bas et en Grande-Bretagne (dans ce dernier cas, touchant la déclassification du cannabis). Pourtant les commissions et comités étaient généralement composés de per- 
sonnes respectables et dont l'opinion scientifique ou morale ou les deux, était difficilement répudiable. Les enquêtes s'étaient souvent étendues sur plusieurs années ou même de nombreuses commissions s'étaient penchées sur le même problème comme c'était le cas aux États-Unis et en Angleterre, et les études avaient coûté plusieurs millions de dollars. À quoi fallait-il donc attribuer l'inertie des parlements ou des gouvernements face aux recommandations de leurs comités et commissions?

Il faut bien constater que des groupes sociaux nombreux et influents ont intérêt, dans chacun des pays, à ce que soit maintenue la prohibition qui touche quelques substances. M'appuyant sur mon analyse de la situation canadienne et des États-Unis, j'énumérerai ces groupes dans l'ordre où ils sont apparus avec leurs intérêts particuliers sur la scène publique des audiences ou dans les corridors du pouvoir politique.

1. Le premier groupe à manifester bruyamment son opposition à toute libéralisation des lois sur les drogues a été la police, les corps policiers de tous niveaux : fédéral, provincial, municipal, les escouades spéciales travaillant dans le domaine des stupéfiants, surtout. Ces dernières, comme on sait, tirent un prestige non négligeable de leurs contacts internationaux et des effets médiatiques de leurs "prises». Le discours des corps de police s'est avéré d'une grande hypocrisie (leurs affirmations à l'effet que la situation était sous contrôle ou pouvait l'être ne se comptent pas) et d'un fondamentalisme moral difficile à imaginer pour ceux qui ne les ont pas entendus. L'influence des corps de police sur les gouvernements n'est plus à démontrer: les ministères de la justice et de l'intérieur, etc. exercent souvent un contrôle particulier sur les autres départements d'État. Le lobby qu'exercent les corps de police au niveau national a des ramifications internationales. Enfin, tous les moyens ont paru bons, aux policiers de certains pays, pour discréditer les tenants de la libéralisation des lois sur les drogues: dossiers montés de toutes pièces, accusations sur la place publique;

2. Le deuxième bastion de résistance au changement était et est toujours composé de médecins: la profession médicale s'était indignée, dans les années soixante, que des jeunes et des citoyens d'apparence douteuse se prétendent capables de distinguer les vertus enivrantes, analgésiques ou euphorisantes de quelques drogues et même découvrent des contre-poisons dans les pharmacies des parents sous forme de valiums, par exemple, en cas de «mauvais voyage». Cette même profession n'a pas consenti à s'intéresser vraiment aux nouvelles questions 
que posaient les usagers. Seul, un très petit nombre de médecins omnipraticiens et quelques psychiatres et pharmacologues, d'ailleurs jugés sévèrement par leurs confrères, sont «descendus dans la rue» et ont aidé les usagers et les travailleurs sociaux à mettre sur pied quelques centres d'information, quelques cliniques d'urgence, des services ambulants. Pour l'ensemble, la profession a nié le problème ou plutôt nié qu'il s'agissait d'une question qui la concernait et renvoyé les usagers au tribunal pénal, contribuant à raffermir les positions des prohibitionnistes;

3. Les pharmaciens se sont montrés particulièrement froissés qu'on puisse se droguer sans leurs conseils et avec des substances n'appartenant pas à leur rayon de compétence. Ajoutons que ce corps professionnel avait des raisons additionnelles de se sentir menacé car une certaine portion des usagers a tenté de détoumer le public de l'usage des «drogues chimiques» au profit des «drogues naturelles»; d'autre part, nombreux sont les usagers et les membres de la contre-culture qui, devant les accusations de nocivité lancées contre les «drogues de rue» ou drogues illégales, ont répliqué en dénonçant les méfaits bien réels des médicaments;

4. Parmi les groupes corporatifs les plus menacés et dont la voix s'est fait entendre sur le registre aigu et les pressions politiques sous forme de chantage, il faut compter les laboratoires de produits pharmaceutiques; les représentants de grandes compagnies, comme Roche, ont tenté de se défendre devant quelques commissions d'enquête qui s'apprêtaient à dénoncer l'effet d'accoutumance des médicaments;

5. Les distilleurs et brasseurs, en plus d'exercer les lobbies que l'on imagine auprès d'hommes politiques qui sont déjà gagnés aux vertus de l'alcool, ont tâché de corriger l'impression d'irresponsabilité qu'ils pouvaient laisser à la population et de faire preuve de bonne volonté : certains ont commencé à prôner la modération... bien entendu en même temps que la consommation!

6. Les compagnies de tabac exercent sur les hommes politiques des pressions comparables. En effet, lorsque les témoins s'attaquaient trop ouvertement aux méfaits de la cigarette, certaines sont venues se défendre en arguant qu'elles avaient beaucoup réduit la teneur en nicotine de leur produit, estimant qu'il est injuste de comparer une drogue entrée dans les moeurs et donc subissant les effets des contrôles sociaux, à des substances dont la nocivité «réelle» est inconnue et l'usage encore marginal. Mais l'argument majeur des compagnies de tabac en est probablement un qui frappe les gouvernements là où ils sont le plus sensibles: de larges secteurs d'emploi disparaîtront si l'on 
diminue la consommation de tabac et si la cigarette de nicotine souffre de la compétition avec, par exemple, une cigarette de cannabis;

7. Enfin, on a vu apparaître devant les commissions nationales chargées d'étudier le problème de la drogue ceux que j'appellerai les entrepreneurs moraux: les responsables des cliniques anti-drogues, et des groupes d'ex-toxicomanes, des groupes de parents, des représentants de plusieurs églises et même des membres ou des représentants de quelques professions sociales (associations de travailleurs sociaux, de psycho-éducateurs, de psychologues, de psychiatres, d'avocats, de magistrats de la jeunesse) clamant leur indignation, leur scandale devant la possibilité qu'on relâche les lois sur les drogues: il fallait à tout prix s'opposer à toute libéralisation.

Tous ces groupes représentent non seulement beaucoup de monde, mais surtout beaucoup d'influence et de pouvoir de pression. En plus de leur capacité de convaincre, les distilleurs, les brasseurs, les laboratoires pharmaceutiques, les compagnies de tabac, disposent de moyens terriblement efficaces de faire pencher les partis politiques du côté qui sert leurs intérêts: les caisses électorales sont en effet souvent redevables à ces entreprises. D'autre part, en ce qui touche aux grandes corporations professionnelles, rares sont les gouvernements qui peuvent se permettre de les indisposer sérieusement.

Ces groupes de pression et d'intérêt, qui se sont exprimés avec force en faveur de la prohibition depuis 10 ou 15 ans, tiendraient-ils le même langage aujourd'hui? Si oui, les États leur prêteraient-ils une oreille aussi attentive? D'autres groupes tenant un langage différent, ouvert à la décriminalisation ou à l'abolition de la prohibition réussiraient-ils à se faire entendre? L'analyse des résistances, pour être sérieuse et probante, doit tenir compte des situations particulières dans chaque pays et les réponses ne seront sans doute pas les mêmes partout. De surcroît, cette analyse doit tenir compte de la pression internationale s'exerçant par les conventions des Nations-Unics.

\section{LES RÉSISTANCES SUR LA SCÈNE INTERNATIONALE}

Voilà déjà plus de 75 ans que les premiers traités internationaux sur les drogues ont été signés (1912): on se rappellera qu'avant cette date, plusieurs pays européens, la France et les Pays-Bas notamment, ne criminalisaient pas l'usage de plusieurs substances maintenant prohibées, tandis que les pays scandinaves et anglosaxons recouraient déjà au droit pénal pour interdire l'usage de plusieurs drogues par le délit de simple possession. 
Depuis 1912, date du premier traité, on a vu sans cesse croître:

1. le nombre de pays signant les ententes internationales sur le contrôle des drogues;

2. le nombre de substances incriminées;

3. le nombre des actes défendus dans les statuts nationaux sur les drogues.

Progressivement, «les appareils de contrôle policier, judiciaire et pénal, dans les pays ayant signé les ententes, se développent et se mettent à échanger; l'objet du contrôle passe du trafic au consommateur; la politique des drogues devient centralisée et émane de plus en plus des Nations-Unies: c'est le modèle de la politique des drogues américaine qui se généralise dans les pays signataires. La Drug Enforcement Administration devient le modèle international des contrôles policiers en matière de drogue» (Hulsman et Van Ransbeek, 1983, 273).

La convention de 1961, en particulier, mais aussi celle de 1972 , constituent maintenant une source de résistance fondamentale aux changements des lois sur les drogues dans les différentes sociétés nationales signataires et même dans les autres, car l'impérialisme américain ou même la pression morale des états membres jouent sur les pays producteurs, à travers ces ententes internationales.

Selon Hulsman et Van Ransbeek, il est extrêmement difficile, pour des groupes de citoyens nationaux, d'être informés correctement de ce qui se passe aux Nations-Unies lors des discussions des grandes conventions sur les drogues. Il est encore plus difficile de se mobiliser pour faire valoir les opinions de groupes, fussent-ils majoritaires. Or il ne devient possible d'influencer le processus législatif international que dans des cas où les groupes de contrepression disposent d'assez de pouvoir et maîtrisent le langage politique international (Ibid. 277).

\section{LES SOLUTIONS DE RECHANGE À LA PROHIBITION}

Sur le diagnostic d'inefficacité et sur le caractère pernicieux de la prohibition, le consensus s'élargit et s'approfondit si l'on en juge par les débats des années 80 . «Les lois sur les drogues font plus de tort que les drogues» (Bertrand, 1989); «tous les problèmes décrits dans la publication de l'O.N.U.: Les Nations-Unies et la lutte contre l'abus des drogues, sont liés directement à l'illégalité des substances et non aux substances en tant que telles» (Sengers, 1988); «Le seul résultat de la 
guerre à la drogue c'est l'inflation des prix dont les trafiquants ont besoin pour s'assurer de la rentabilité de leur commerce» (Grinspoon et Bakalar, 1988); «La drogue n'est pas interdite parce qu'elle est dangereuse; elle est dangereuse parce qu'elle est interdite (Apap, 1988); «L'interdiction (...) a eu pour effet de nuire à ceux qu'elle essayait de protéger et a en même temps fait peser un énorme poids social et économique sur le reste de la société. Vu la gravité de la situation actuelle, la légalisation mérite d'être prise en considération comme une alternative radicale» (Stevenson, 1988). «Malheureusement la guerre à la drogue a offert et continue à offrir à l'homme moderne une grande partie de ce qu'il semble désirer le plus: une fausse compassion et une authentique coercition, une pseudo-science et un réel paternalisme, des fausses maladies et des traitements métaphoriques ainsi que des politiques opportunistes et une hypocrisie onctueuse. Il m'est difficile de comprendre comment quiconque ayant des notions d'histoire, de pharmacologie (...) comment ces personnes peuvent éviter d'en conclure que la guerre à la drogue est tout simplement un nouveau chapitre de l'histoire naturelle de la stupidité humaine» (Szasz, 1988).

Les auteurs sont aussi très généralement d'accord sur la difficulté de renverser le mouvement: les Européens sont particulièrement sensibles au caractère définitif et fermé des conventions internationales.

1. Pourtant, plusieurs proposent d'instaurer, en lieu et place de la prohibition qui a fait ses preuves d'inefficacité et d'effets pervers, un système de licence, étatique, qui exerce un contrôle sur l'approvisionnement, la qualité, la circulation des drogues illicites.

Cette transformation doit se faire sur une grande échelle nationale et peut-être internationale si on veut éliminer les risques que perdure le commerce illicite et que tous les malheurs associés à celui-ci se continuent.

2. La tiajorité estime que toutes les drogues doivent être soumises au même contrôle que le tabac et l'alcool.

3. Certains y vont de propositions plus précises et originales. C'est le cas notamment de Grinspoon qui recommande un impôt sur la nocivité.

Je voudrais proposer ici un exercice utopique. (...) Il s'agit de la légalisation et de la fiscalisation des substances contrôlées actuellement. Les taxes serviraient à financer des programmes nationaux d'éducation en matière de stupéfiants ainsi que les frais de sécurité sociale liés aux abus. Une commission nationale serait désignée 
pour établir une taxe sur les stupéfiants fondée sur le coût social de chaque substance. Le taux d'imposition serait alors ajusté d'année en année selon les données les plus récentes en la matière. Ces données ne sont peut-être pas disponibles à l'heure actuelle, mais grâce aux techniques modernes de récolte et de traitement des données, le processus est vraisemblable. Le gouvernement pourrait ainsi reconnaître ceux qui seraient d'inévitables victimes de la drogue et chercherait à les orienter vers les substances moins nocives à l'aide de la fiscalisation et du programme d'éducation. Avec ce système aucune distinction ne serait faite entre les drogues légalement tolérées actuellement, comme l'alcool et le tabac, et les autres stupéfiants». (Grinspoon et Bakalar, 1988).

Dans un deuxième temps cependant et à la réflexion, l'auteur estimait plus réaliste de limiter cette expérience à deux drogues : le tabac et le cannabis.

4. De leur côté, les représentants hollandais, certains en tout cas, font valoir que leur expérience de décriminalisation des drogues douces et d'ouverture et de tolérance à l'endroit des toxicomanes a singulièrement assaini la situation.

5. Quant à la Fondation pour la politique des drogues (Washington), elle propose quatre types d'action visant: 1) à rendre possible l'utilisation de la marijuana comme médicament; 2) à protéger les citoyens contre les fouilles et examens abusifs; 3) à faire en sorte que les toxicomanes puissent être traités avec drogues autant que sans drogue; 4) à réduire les dangers que courent les corps de police, en obtenant des gouvernements que des compromis humanitaires viennent mettre une sourdine à la guerre à la drogue :

«The Drug Policy Foundation is an international group of distinguished scholars, professionals, corporate executives and concerned citizens dedicated to the search for responsible drug-law reform. Here is a short list of issues on which we are working:

Medicine for the Sick - There is no scientific or technical reason why the government denies heroin and marijuana to sick people suffering from cancer, glaucoma, multiple sclerosis and other diseases. These drug should be made available to ease the suffering of millions of citizens. The Drug Policy Foundation is participating in a suit against the government to allow doctors to prescribe marijuana for their patients who need it.

Protection for Citizens' Rights - Mass random urine testing and other extreme drug-war measures are destroying the rights and dignity of millions of people, most of whom have no involvement in illicit drugs. The Drug Policy Foundation is working to protect your right against unreasonable search and seizure. 
Drug Treatment - Another perversity of the drug war is the fact that addicts often must wait months before receiving treatment. The Drug Policy Foundation believes that addicts should receive treatment on demand, clean needles and medicinal drugs under doctors' supervision, as well as drug-free treatment.

Protection for Police - Our police are victims of the drug war. The huge profits created by our drug laws create irresistible temptations for many good officers. The vicious criminals attracted to the rich black market represent a constant threat of violence to them. Only by seeking humane compromises in the drug war will the erosion of police institutions be halted.»

Bien que chacune des solutions se heurte à des obstacles considérables, il n'a semblé à aucun des participants aux conférences de Londres, Bruxelles et Washington qu'il convenait de baisser les bras, de laisser faire la guerre à la drogue. Tous croyaient que la prohibition avait assez duré.

\section{BIBLIOGRAPHIE}

APAP, G. (1988), La persécution des drogués relève-t-elle d'une idéologie? Colloque sur l'Antiprohibitionnisme, Bruxelles, octobre 1988. Non publié.

BERTRAND, M.A. (1973), Conclusions et recommandations supplémentaires. Rapport final de la Commission d'enquête sur l' usage des drogues à des fins non-médicales. Ottawa, Information Canada, pages 229-241.

BERTRAND, M.A. (1984), Le contrôle de la drogue au Canada dix ans après LeDain. Le Devoir, 26-03-84, p. 14-15.

BERTRAND, M.A. (1986), La politique des drogues. Permanence des effets pervers et résistance au changement des lois sur les drogues. Déviance et Société, 10, 2, p. 177-193.

BERTRAND, M.A. (1989), L'immoralité de la prohibition. Nouvelles radicales, janvier, p. 13-16.

BLACKWELL, J. (1988), An Overview of Canadian Illjcit Use Epidemology, in : Hicit Drugs in Canada. a Risky Business. J.C. Blackwell et P.G. Erikson ed, Scarborough, Ontario, Nelson Canada.

DRUG POLICY FOUNDATION (1988), Programme de la Conférence internationale sur la réforme de la politique des drogues. Washington, 20-23 octobre 1988, 4 pages.

GRINSPOON, L.; BAKALAR, J.B. (1988), L'impôt sur la nocivité : une proposition de réglementation par l'impôt sur les stupéfiants. Colloque sur l'Antiprohibitionnisme, Bruxelles, octobre 1988. Non publié.

HULSMAN, L.; VAN RANSBEEK, H. (1983), Évaluation critique de la politique des drogues. Déviance et Société, 7, 3, 271-280.

LeDAIN, G., prés., (1973), Rapport final. Commission d'enquête sur l'usage des drogues à des fins non-médicales. Ottawa. Information Canada. 
NEGRETE, J.C., (1988), Les conséquences de l'usage régulier de cannabis. «Bilan et perspectives nouvelles». Conférence d'action nationale sur l'usage des drogues. Montréal, 26-29 octobre. Non publié.

SENGERS, W. (1988), Les Nations-Unies et la lutte contre l'abus des drogues. Prémisses, commentaires et conclusions. Colloque sur l'Antiprohibitionnisme, Bruxelles, octobre 1988. Non publié.

SINGLE, E., (1988), The impact of marijuana decriminalization : an update. Paper presented at The National Action Conference on Drug Abuse. Montreal, October 1988. Unpublished.

STEVENSON, R.C. (1988), Arguments économiques en faveur de la légalisation des stupéfiants. Colloque sur l'Antiprohibitionnisme, Bruxelles, octobre 1988. Non publié.

SZASZ, Thomas (1988), Plaidoyer pour la fin de la guerre la plus longue du XXe siècle - la guerre à la drogue. Colloque sur l'Antiprohibitionnisme, Bruxelles, octobre 1988. Non publié. 